1970. Mortality inequalities continue to exist between population sub-groups.

Methods Ecological study using data about mortality of the Improvement Program of Information on Mortality of São Paulo and population estimates by demographic census (IBGE-2000). Areas were drawn from the Social Inclusion Map for the City. The outline was based on territorial classification of the 96 administrative districts in five areas, according to the index of social districts: Areas 1 and 2 (inclusion) and 3, 4, 5 areas (exclusion). We examined cardiovascular mortality rates calculated for 3-year averages age-standardised, relative to differences and rates ratio between areas (CI of 95\%).

Results Cardiovascular mortality declined in all areas and both male and female sexes in these periods. It was observed major decline (30\%) between 1996-1998 and 2003-2005 in rich areas and it was smaller in poor areas (5\%). The highest differential was among male, with rate ratio (A5/A1) that it changed from 1.02 (95\% CI 0.99 to 1.05 ) in first period for 1.38 in the last period ( $95 \%$ CI 1.34 to 1.42$)$. In the women this ratio changed from 0.79 (95\% CI 0.77 to 0.81 ) for 1.07 (95\% CI 1.03 to 1.09 ).

Conclusion Although overall decline in cardiovascular mortality in all socioeconomic status, it was observed increasing of the inequality in reduction of this death rates, which may reflect worsening living conditions or less access to the health services and to the development diagnostic and therapeutic methods.

\section{SP1-100 SURVIVAL ANALYSIS AND RISK FACTORS FOR VALVE SURGERY IN BRAZILIAN CHILDREN AND ADOLESCENTS WITH RHEUMATIC HEART DISEASE}

doi:10.1136/jech.2011.142976n.77

\begin{abstract}
${ }^{1,2} \mathrm{R}$ E Müller, ${ }^{*}{ }^{2} \mathrm{M}$ V M Peixoto, ${ }^{2} \mathrm{~L}$ Higa, ${ }^{3} \mathrm{M}$ Cunha, ${ }^{1} \mathrm{R}$ Xavier, ${ }^{1,4} \mathrm{M} \mathrm{C}$ Kuschnir. ${ }^{1}$ National Cardiology Institute (INC), Rio de Janeiro, Brazil;, ${ }^{2}$ Fernandes Figueira Institute (IFF)/FIOCRUZ, Rio de Janeiro, Brazil; ${ }^{3}$ National Public Health School, FIOCRUZ, Rio de Janeiro, Brazil; ${ }^{4}$ State University of Rio de Janeiro, Rio de Janeiro, Brazil
\end{abstract}

Introduction Rheumatic heart disease (RHD) is still prevalent in developing countries, with 332.000 annual deaths estimated worldwide. In Brazil, RHD remains a major problem, responsible for high mortality/morbidity and great social impact, with many patients requiring surgical intervention during childhood. Our aim was to investigate the time and risk factors leading to valve surgery in children and adolescents with RHD in a tertiary center in Rio de Janeiro.

Methods Data were reviewed on 137 patients followed-up between 1988 and 2007. RHD diagnosis was assigned with revised Jones criteria and/or Doppler-echocardiography indicating chronic mitral/aortic lesions. Kaplan-Meier method was used to determine time until surgery and Cox model to evaluate potential risk factors: age; gender; clinical status (acute; recurrence; chronic heart disease); recurrence rate; secondary prophylactic status and endocarditis.

Results The studied population was $52.5 \%$ female, median age 11.2 years. Median follow-up time 7.3 years. The proportions were $33.5 \%$ for non-adherence to prophylaxis, $31.4 \%$ for recurrence rate, $8.8 \%$ for endocarditis where most underwent surgery (83.3\%). Surgical rate (41\%) was different between male (53.8\%) and female (29.2\%). Survival curves showed most events on the first 2 years and none after 10 years. Cox analysis confirmed the male gender and endocarditis as significant factors.

Conclusion Brazilian RHD children and adolescents committed with endocarditis suffered more risk of having valve surgery. Although most patients underwent surgery in first 2 years, long-term surveillance of this population is necessary because of possible reintervention. Survival analysis for repetitive events should be applied to the evaluation of recurrent episodes and reoperation.

\section{SP1-101 A MODEL OF INTERNATIONAL MULTI-SITE COLLABORATION FOR CHRONIC DISEASE RESEARCH IN DEVELOPING COUNTRIES}

doi:10.1136/jech.2011.142976n.78

${ }^{1} \mathrm{H}$ Z Qian, ${ }^{* 2} \mathrm{~N}$ Dianis, ${ }^{2} \mathrm{~J}$ Bolognese. ${ }^{1}$ Vanderbilt University, Nashville, Tennessee, USA; ${ }^{2}$ Westat, Rockville, Maryland, USA

The impact of chronic diseases continues escalating among populations in developing countries. In an effort to combat chronic cardiovascular and lung diseases globally, NHLBI and the United Health Chronic Disease Initiative support local research and training capacity building programs for a network of 11 collaborating Centers of Excellence (COEs) in low- and middle-income countries. Each COE plans to implement1-4 projects; 20 projects are research-related [four focus on pulmonary diseases, eg, chronic obstructive pulmonary disease (COPD), and 19 focus on cardiovascular diseases (CVD), eg, hypotension and stroke] and others provide training, community outreach service, and institutional capability building opportunities. Six projects are conducted in multiple countries, involving 21 developing countries. Studies typically target adults, though two studies target children and adolescents. Projects adhere to the following study designs: qualitative assessment and focus group discussion, case-control, crosssectional, prospective cohort, quasi-intervention, randomised clinical trial, and simulation. COEs primarily collect data on sociodemographics; anthropometry and blood pressure; lifestyle such as smoking, alcohol use, and physical activities; medical history and medication use; and nutrition. For other studies, COEs collect data on mental health, quality of life, health service utilisation, female reproductive history, spirometry, and biomarkers of CVD and pulmonary diseases. Collecting similar types of data may allow cross-study and cross-national data analysis. Each developing country is paired with at least one academic partner from a developed country to enhance sustainable research and training activities to tackle the complex challenges of the CVD and pulmonary non-communicable chronic disease burden.

\section{SP1-102 ORAL STATUS AND ITS ASSOCIATION WITH UNDERWEIGHT AND OVERWEIGHT/OBESITY IN BRAZILIAN INDEPENDENT-LIVING OLDER PEOPLE}

doi:10.1136/jech.2011.142976n.79

${ }^{1} \mathrm{~L}$ H do Nascimento Tôrres, ${ }^{*}{ }^{2} \mathrm{D} D$ da Silva, ${ }^{3} \mathrm{~A}$ L Neri, ${ }^{4} \mathrm{~J}$ B Hilgert, ${ }^{4} \mathrm{~F}$ N Hugo, ${ }^{1} \mathrm{M}$ da Luz Rosário de Sousa. ${ }^{1}$ Faculdade de Odontologia de Piracicaba, Piracicaba/São Paulo, Brazil; ' Universidade de São Paulo, São Paulo, Brazil; ${ }^{3}$ Universidade Estadual de Campinas-UNICAMP, Campinas/São Paulo, Brazil; ${ }^{4}$ Universidade Federal do Rio Grande do Sul, Porto Alegre/Rio Grande do Sul, Brazil

Introduction Evidence suggests that tooth loss may lead to changes in food choice due to eating difficulties. The relationship between use of dental prosthesis and changes in body mass index among elderly is still unclear. This study aimed at assessing the association between oral rehabilitation, self-perceived chewing ability and nutritional status in Brazilian elderly.

Methods This study is part of a major project-the FIBRA studycarried out in Campinas, Brazil. The sample of this cross-sectional study was composed by 900 independent-living older people. Complete data were available for 545 persons. Dental prosthesis was assessed in accordance with the WHO criteria. Height and weight were used to generate body mass index (BMI) data. The data regarding dental prosthesis use and self-perceived limitation in the type or amount of food intake due to problems with prostheses or their lack generated a new variable, oral status. Participants were categorised into eutrophic, underweight or 